\title{
Eosinophilic granulomatosis with polyangiitis (Churg-Strauss syndrome) masquerading as acute coronary syndrome
}

\author{
Authors: Peter Moore ${ }^{A}$ and Lin Thiri Toon ${ }^{A}$
}

\section{Case presentation}

A 74-year-old man with a history of ankylosing spondylitis presented with a 5-day history of feeling generally unwell. He complained of burning retrosternal chest pain, cough productive of white sputum, shortness of breath, confusion, fevers and fatigue. He also mentioned seeing transient flashing lights. On examination, he had bibasal crackles on auscultation and splinter haemorrhages on his fingernails and toenails. Investigations revealed raised inflammatory markers, a raised white cell count (notably with a high eosinophilia), a raised troponin and D-dimer. Electrocardiography (ECG) showed incomplete right bundle branch block with left axis deviation and a chest X-ray was normal.

Initially, the patient was treated according to the acute coronary syndrome (ACS) protocol and was transferred to the coronary care unit. Following admission, he was investigated for infective endocarditis, but serial blood cultures were negative and echocardiography found no vegetations. Further investigations excluded parasitic infections and autoimmune conditions. The patient later developed slurred speech and decreased coordination. Computed tomography (CT) of his head showed a small low attenuation area in the right superior frontal lobe, reported as likely recently established ischaemic infarcts. $\mathrm{CT}$ of his chest/abdomen/pelvis excluded localised infections and occult malignancy, but showed a small pericardial effusion, pre-existing ankylosing spondylitis and bibasal pulmonary collapse/consolidation. Autoimmune screening showed a weak/ moderately positive result for perinuclear anti-neutrophil cytoplasmic antibodies (pANCA) and a raised IgG. The diagnosis was eosinophilic granulomatosis with polyangiitis (EGPA) and the patient was treated with pulsed doses of intravenous methylprednisolone followed by oral prednisolone.

\section{Background}

EGPA (Churg-Strauss syndrome) is a rare autoimmune antineutrophil cytoplasmic antibody (ANCA)-associated vasculitis. According to the Revised International Chapel Hill consensus conference nomenclature of vasculitides (2012), it is defined as an eosinophil-rich and necrotising granulomatous inflammation often involving the respiratory tract, and necrotising vasculitis predominantly affecting small- to medium-sized vessels, associated with asthma and eosinophilia. ${ }^{1,2}$ The condition is a multisystem

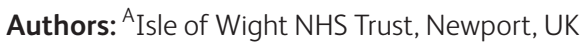

disorder and therefore often has non-specific presentations and multi-organ involvement. Typically, it has three stages of symptoms: the allergic stage, the eosinophilic stage and the vasculitic stage. These phases do not always occur sequentially and individuals may not develop all three phases. Phases last from 6 months to two years. ${ }^{3}$ There are few described cases of EGPA with cardiovascular presentations; manifesting as an ACS, acute myocarditis and cardiogenic shock, isolated cardiac tamponade, eosinophilic endomyocarditis with rapidly progressive diastolic dysfunction, the formation of apical thrombosis of both ventricles and ventricular tachycardia. ${ }^{4}$ The Lanham criteria and the American College of Rheumatology criteria are used for diagnosis, but blood tests are diagnostically useful. pANCA is positive in $40-60 \%$ of cases, eosinophilia is common but transient and elevation of IgG4 is also common. ${ }^{5,6}$ Treatment involves corticosteroids and cyclophosphamide (for remission induction), azathioprine and methotrexate (for remission maintenance) and B-cell depletion with rituximab has shown promising results for remission induction. ${ }^{1}$

\section{Conclusion}

EGPA is a rare autoimmune condition with non-specific presentations and multi-organ involvement. Eosinophilia, raised inflammatory markers and raised pANCA can be the clues for diagnosis, but raised troponin and D-dimer tests are sometimes misleading.

\section{Conflicts of interest}

None declared.

\section{References}

1 Jennette JC. Overview of the 2012 revised International Chapel Hill Consensus Conference nomenclature of vasculitides. ClinExp Nephrol 2013;17:603-6.

2 Gioffredi A, Maritati F, Oliva E, Buzio C. Eosinophilic granulomatosis with polyangiitis: an overview. Front Immunol 2014;5:549.

3 National Organization for Rare Disorders. Churg Strauss syndrome. NORD, 2019. https://rarediseases.org/rare-diseases/churg-strausssyndrome [Accessed 31 October 2019].

4 Budanova M, Mitrofanova L, Kozlenok A et al. Ventricular tachycardia as the first manifestation of Churg-Strauss syndrome. $J$ Cardiol Cases 2017;15:61-4.

5 Furuta S, Iwamoto T, Nakajima H. Update on eosinophilic granulomatosis with polyangiitis. Allergol Int 2019;68:430-6.

6 Science.gov. Sample records for background Churg-Strauss syndrome. Science.gov, 2019. www.science.gov/topicpages/b/background+ churg-strauss+syndrome[Accessed 31 October 2019]. 